\title{
Image Enhancement through intensity of edge pixels using Honey Bee Mating Optimization
}

\author{
${ }^{1}$ Kapila Molri, ${ }^{2}$ Sachin Gupta \\ ${ }^{1}$ Fellow, \\ ${ }^{2}$ Assistant Professor SRMIET, Kohra-bhura, Narayangarh, Haryana, India,
}

\begin{abstract}
There are many techniques of image enhancement as well as optimizing the same process in the image processing such as Powell's method, Fast Ostu's method, Histogram equalization (HE) method, Particle swarm optimization (PSO) and Honey bee (HB) algorithm.

In this paper, a new approach to automatic image enhancement using honey bee mating optimization is implemented by specifying intensity of the edges pixels and also earlier reported PSO results were used. Further results of previous work i-e PSO and HBMO are compared through different parameters. The obtained results indicate that the proposed Honey bee algorithm yields better results in the terms of both the maximization of number of the pixels in the edges and peak signal to noise ratio (PSNR). Computational time is also relatively small in the Honey bee enhanced image as compared to the PSO case which is earlier reported.
\end{abstract}

Keyword: Swarm optimization, honey bee mating optimization and image enhancement.

\section{Introduction}

Digital image basically consists of an array of digital count values with each value representing the brightness, or gray level of a pixel in the image. Image processing improves the efficacy of the image data. Digital image enhancement is basically process that transforms the input image to the better image by improving the interpretability of information in images .It accentuates or sharpens image features such as edges ,boundaries or contrast to make a graphic display more meaningful for analysis.

This enhanced image can be provided as the input for the other automated image processing technique and applications. Inherent information content of the image doesn't get increased by enhancement method rather the dynamic range of the chosen features is increased. In image enhancement here many techniques are available for the enhancement of an image. Those techniques are histogram equalization (HE), genetic algorithm (GE), particle swarm optimization (PSO) [1], [4] honey bee (HB).

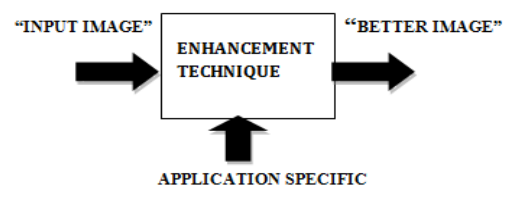

Fig1. Enhancement Model

Swarm intelligence has become a research interest to many research scientists of related fields in recent years. Bonabeau has defined the swarm intelligence as "any attempt to design algorithms or distributed problemsolving devices inspired by the collective behavior of social insect colonies and other animal societies" [1].

Boabeau et al. focused their viewpoint on social insects alone such as termites, bees, wasp as well as other different ant species.

However, the term swarm is used in a general manner to refer to any restrained collection of interacting agents or individuals. The classical example of a swarm is bees swarming around their hive; nevertheless the metaphor can easily be extended to other systems with a similar architecture. An ant colony can be thought of as swarm of birds. An immune system [2] is a swarm of cells and molecules as well as a crowd is swarm of people [3]. Particle Swarm Optimization (PSO) Algorithm models the social behavior of bird flocking or fish schooling [4].Many authors exposed the use of PSO to solve variety of problems in computer science and engineering $[4,5]$. In this paper, author used HB based enhancement method on medical image for tumor detection. Further results obtained after applying HB method and earlier reported results using PSO were compared.

\section{Proposed Enhancement Model}

There are key steps when applying HB to optimization problems:

- Place the hive of bees at the centre of any place.

- Here 1000 bees are initialized; all these bees spread from the hive for find the food. 
- When any bee is employed (knowledge of food), that employed bee came back to the hive by making the path from food place to hive with making white edges.

- If "yes" then after coming back to the hive, the employed bees spread randomly and follow a new path which is unemployed.

- If predefined value is less than the numbers of bees are returns to next then OK.

- If "yes" then proc3ess is complete.

- But if "NO" process is not done then this process is again start on the step 3.

The flow chart brief the steps followed during the algorithm implementation.

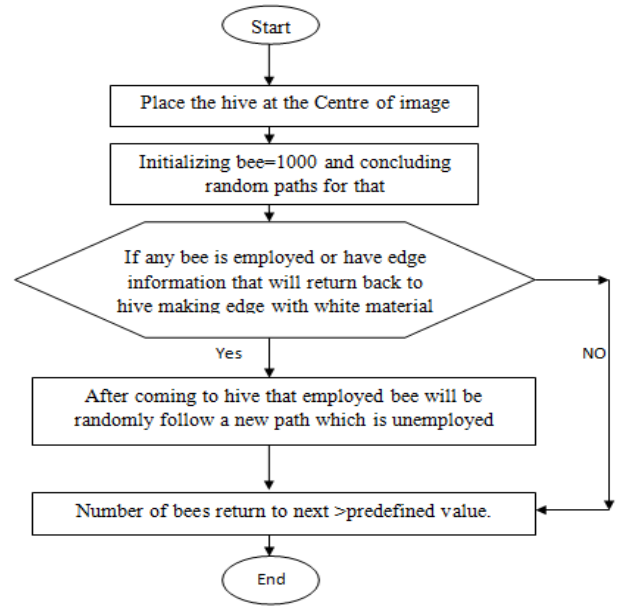

The proposed work optimizes edge intensity pixels using Hybrid honey bee .In the algorithm random paths are chosen for the bees with their initial position at the center of the hive. Here in this algorithm PSO based search phenomenon is used to find edges i-e neighborhood search phenomenon is used. Fitness function or value is basically the number of edges found. If any bee is having edge information that will return back to hive making edge with white material. A honey -bee colony consists of queen(s) (best solution), drones(incumbent solutions), worker(s) (heuristic), and broods(trial solutions). The HBMO algorithm simulates the natural mating behavior of queen bee when she leaves the hive to mate with the drones .After each successful mating, the drone's sperm is added to the queens spermatheca .Before the mating flight the queen is initialized with some energy and only ends her mating flight when her energy level drops below a threshold( which is close to zero) .Tablel illustrates the analogy between the natural honey bee colony and the artificial honey bee algorithm.

Table1 - Analogy between the natural honey bee colony and the artificial honey bee algorithm

\begin{tabular}{|l|l|}
\hline NATURAL HONEY BEE & ARTIFICIAL HONEY BEE \\
\hline QUEEN & BEST SOLUTION \\
\hline DRONES & INCUMBENT SOLUTIONS \\
\hline BROODS & NEW TRIAL SOLUTIONS \\
\hline WORKER & HEURISTIC SEARCH \\
\hline MATING,BREEDING & CROSS OVER \\
\hline
\end{tabular}

The queen mate probabilistically, using equation (Abbass2001a )

$$
\mathrm{p}(\text { Queen,Drone } \mathrm{i})=\mathrm{e}\left[-\Delta \mathrm{f} / \text { energy }_{(\mathrm{t})}\right]
$$

Where $\mathrm{p}$ (Queen,Drone) represents the probability of accepting the $\mathrm{i}^{\text {th }}$ for mating. $\Delta(\mathrm{f})$ represents the absolute fitness difference between the drone and queen i-e $\Delta(\mathrm{f})=\mid \mathrm{f}($ queen $)-\mathrm{f}($ drone $) \mid$,energy(t) refers to the queen's energy at the time $t$ of mating. The queen moves between different states (i-e solutions) in the allocated space according to its energy mates with the drones using equation above. Once a drone has mated with queen its sperm is added to queen's sperm theca. The queen ends her mating flight when her energy level drops below threshold (which is close to zero) or the queen's maximum sperm theca size is reached. At the end of mating flight, the queen returns to the nest. In our research work we had eliminated the speed parameter since it didn't affect the selection of drone to mate with the queen. If the mating is successful (according to the probabilistic decision rule), the drone sperm is added into the queen's spermatheca.

\section{Parameters defined}

We initialize three user defined parameters

- Number of queens.(bees=1000)

- The queen's spermatheca size, which represents the maximum number of mating each queen, performs in single mating flight, thus also the number of broods that will be born after each mating flight. And 
- The number of workers. We only use one worker (heuristic search)i-e descent algorithm.

\section{Results and Discussion}

The optimization problem considered in this paper is to solve the enhancement problem using HBMO. Our objective is to maximize the number of pixels in the edges, increase the overall intensity of the edges and to determine PSNR between new developed algorithm and earlier reported method. In order to evaluate the Honey bee algorithm based enhancement method, we have compared the proposed method with PSO-based enhancement method.

For each Honey bee algorithm or PSO run we report three values:

- The performance of the algorithms by comparing the objective evaluation functions in terms of PSNR values.

- The computational time per run of each algorithm.

- The efficiency in terms of the number of edges with gives as indication of the performance of proposed algorithm.

- The fitness value using honey bee (HB) is more when compared with the fitness value using PSO for the same number of generations.

- The computational time for PSO based enhancement was found 94.786 seconds whereas the time taken for HB based enhancement was found 10.785 seconds.

- The computational time is less in case of HB when compared with that of PSO.

- The image that contains the highest number of edges pixels can be rated as having high detail content as shown in Table 2 and Table 3.

- One parameter here used peak signal and noise ratio (PSNR), here after calculate the PSNR.

\begin{tabular}{|c|c|c|c|c|}
\hline Image/fitness & GA & & PSO & HB \\
\hline Cameraman & 102.988 & & 128.821 & 131 \\
\hline Tire & 130.30 & & 136.398 & 146 \\
\hline \multicolumn{5}{|c|}{ Number of edges detected } \\
\hline Image & Original & GA & PSO & HB \\
\hline Cameraman & 2458 & 2575 & 2674 & 3105 \\
\hline Tire & 1823 & 1917 & 2020 & 2836 \\
\hline
\end{tabular}

From figure 2, it is observed that the Honey bee algorithm method yields better quality of solution. In this figure the brightness and contrast of enhanced image using PSO and $\mathrm{HB}$ appear visible. Also, it is clearly visible that brightness of enhanced image using HB is better than brightness of the enhanced image using PSO.

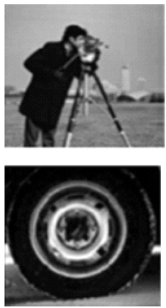

(a)
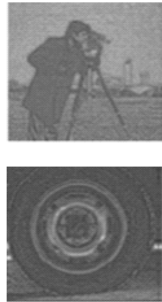

(b)
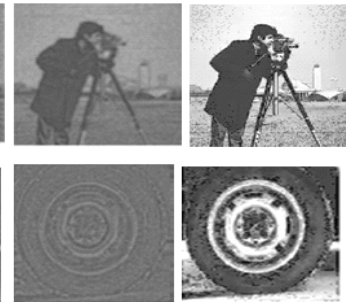

(c)

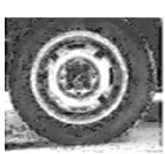

(d)

Fig 2. Enhancement results for the images Cameraman and Tire: (a) left-original images; (b) GA based method; (c) PSO based method, (d)honey bee.

\section{Conclusions}

In this paper, a new optimization algorithm based on intelligent behavior of honey bee swarm has been described. The new swarm algorithm is very simple and very flexible when compared to the existing swarm based algorithms. The objective of the algorithm was to maximize the total number of pixels in the edges thus being able to visualize more details in the images. The algorithm is tested on medical images for tumor detection. The results obtained are tabulated and compared with the results earlier reported using PSO. It is clear from the obtained results that the proposed HB based image enhancement is better than the PSO based image enhancement in terms of quality solution and computational efficiency. The proposed HB based image enhancement method may be extended in several ways, such as: fine turning of the HB parameters in order to reduce the maximum number of iterations.

\section{References}

[1] J. Kennedy, R.C. Eberhart, and Y.Shi, Swarm Intelligence, Morgan Kanufmann Publishers, San Francisco, 2001.

[2] J. Kennedy and R. C. Eberhart, "Particle swarm optimization", Proc. of IEEE International conference on Neural Networks (Perth, Australia), vol. 5, no. 3, pp. 1942-1948, 1995.

[3] Braik,M., "Image enhancement using particle swarm optimization", Proc. of the world Congress on Engineering, Vol. 1, July 2007. 
[4] Change et.al, "Image contrast enhancement based on a histogram transformation of local standard deviation", IEEE Trans Med Imaging, Vol.4, pp.518-31,17, August 1998.

[5] Burdick, H.E, "Digital imaging Theory and applications", Operations or functions on image, Mc Graw - Hill, 1997.,

[6] Chen Wei Fang Kangling , "Multilevel Thresholding Algorithm Based on Particle Swarm Optimization for Image Segmentation", Proceedings of the 27th Chinese Control Conference July 16-18, 2008.

[7] Dah-Chung Chang and Wen-Rong Wu, "Image Contrast Enhancement Based on a Histogram Transformation of Local Standard Deviation", IEEE Transactions on medical imaging, vol. 17, Issue No.4, pp.518-531, August 1998.

[8] M.A. Talal and A.A. Mohamed, " Simulation-based optimization for repairable systems using particle swarm algorithm," Proceedings of the Winter Simulation Conference, Department of Statistics and Operations Research Kuwait University, 2005.

[9] Te-Jen Su et.al, "Particle Swarm Optimization for Gray-Scale Image Noise Cancellation", International Conference on Intelligent Information Hiding and Multimedia Signal Processing, pp. 1459-1462, 2008

[10] Kennedy, J. And Eberhart, R. C., "Swarm Intelligence", Morgan Kanufmann Publishers, , pp. 69-73, 2001.

[11] Fledelius, W. And Brian H. Mayoh, "A swarm based approach to medical image analysis", Proceedings of the 24th IASTED international conference on Artificial intelligence and applications,pp. 150-155, 2006.

[12] F.Maes et.al, "Multimodality image registration by maximization of mutual information," IEEE Trans. Med.Imaging, vol. 6, pp. 8798, no. 2, 1997.

[13] J. P. W. Pluim et.al, “ Mutual information- based registration of medical images-A survey,” IEEE Trans. Med. Imaging, vol. 22, pp. 986-004, no. 8, 2003.

[14] Www.cse.secs.oakland.edu

[15] Dervis Karaboga, “An Idea Based on honey bee swarm for numerical optimization”, Erciyes University, Oct 2005

[16] Phooi Yee Lau et.al,"The detection and visualization of brain tumors on T2-weighted MRI images using multi parameter feature blocks", IEEE,(2005).

[17] Umbaugh Scot E, “Computer Vision and Image Processing”, Prentice Hall, NJ, 1998, ISBN 0-13-264599-8.

[18] R.C. Gonzales, R.E. Woods, "Digital Image Processing”, 2 -nd Edition, Prentice Hall, 2002.

[19] Bailet et.al, "Positron emission tomography: A new precise modality for detection of primary head and neck tumors and assessment of cervical adenopathy," Laryngoscope, vol. 102, pp.281-288, 1992

[20] Abramson C.I et.al, "Effect of insecticides on learning in the Africanized honey bee (Apis mellifera L."- Archives of Environmental Contamination and Toxicology, 37 ,pp 529-535.

[21] Aaron Lefohn, et.al, “Interactive GPU-Based level sets for 3D Brain Tumor Segmentation”, April 16, 2003.

[22] B. Amiri and M. Fathian, "Integration of self organizing feature maps and honey bee mating optimization algorithm for market segmentation", Journal of Theoretical and Applied Information Technology, 2007, pp. 70-86.

[23] Eberhart, R. C. and Kennedy, "A new optimizer using particle swarm theory", Proceedings of the sixth international symposium on micro machine and human science. IEEE, pp. 39-43, 1995.

[24] Eberhart, R. C. And Shi, Y, "Particle swarm optimization: developments, applications and resources", Proceedings of congress on evolutionary computation, IEEE, 2001.

[25] J. N. Kapur et.al, "A new method for gray-level picture thresholding using the entropy of the histogram", Computer Vision Graphics Image Processing, vol-29, , pp. 273-285, 1985

[26] N. Ostu, “A Threshold selection method from gray-level histograms", IEEETransactions on Systems, Man, Cybernetics, SMC-9, , pp. 62-66,1979.

[27] P.S. Liao et.al, “A fast algorithms for multilevel thresholding”, Journal of Information Science and Engineering, 17, pp. 713-727, 2001.

[28] www.jamuimaginecentre.com

[29] www.kingholmes.photoshelter.com

[30] www.jnnp.bmj.com

[31] www.consmicvisoionsworkshop.com

[32] www.neuroadiologycases.com

[33] www.flicker.com

[34] www.bristol.ac.uk

[35] www.hsc.com.my 